\title{
Acute Cerebral Infarction as a Rare Thrombotic Event in Myelodysplastic Syndrome: A Case Report
}

\author{
Hyun Woo Bae, MD, Hyun Dong Kim, MD, PhD, Sung Won Choi, MD, Nami Han, MD, PhD, Mi Ja Eom, MD
}

Department of Physical Medicine and Rehabilitation, Inje University Busan Paik Hospital, Inje University College of Medicine, Busan, Korea

Myelodysplastic syndrome (MDS) is a bone marrow failure syndrome characterized by cytopenia that results in high risks of infection and bleeding. However, there are few reports of cerebral infarction in MDS. We reported a 72-year-old female with MDS who developed acute cerebral infarction. Clinical history of the patient revealed no definite risk factors for stroke except diabetes mellitus and dyslipidemia that was well controlled. This case represented the rare occurrence of arterial thrombosis causing acute cerebral infarction in MDS, which may be due to complex chromosomal abnormality and inflammatory processes.

Keywords Myelodysplastic syndromes, Stroke, Thrombosis

\section{INTRODUCTION}

Major pathogenesis of cerebral infarction includes large vessel thrombosis, cardioembolism, and small vessel occlusion. Myelodysplastic syndrome (MDS) is a bone marrow failure syndrome characterized by cytopenia with high risks of infection and bleeding, which can progress to acute myeloid leukemia [1]. The risk of thrombotic event is relatively low due to cytopenia.

Deep vein thrombosis (DVT) and pulmonary thromboembolism in MDS reportedly occur with concomitant use of lenalidomide and erythropoietin stimulating agents [2].
However, arterial thrombosis especially cerebral infarction in MDS is not previously reported. We described a rare case of acute cerebral infarction in a MDS patient.

\section{CASE REPORT}

A 72-year-old female was admitted to our hospital with acute onset dysarthria and right hemiparesis. The patient was a non-smoker and had no cardiovascular disease. Two years prior, she was diagnosed with MDS of the refractory anemia with excess blasts-2 subtype, according to World Health Organization classification. The

Received April 8, 2016; Accepted May 11, 2016

Corresponding author: Nami Han

Department of Physical Medicine and Rehabilitation, Inje University Busan Paik Hospital, 75 Bokji-ro, Busanjin-gu, Busan 47392, Korea. Tel: +82-51890-6295, Fax: +82-51-891-1430, E-mail: rehabit@inje.ac.kr

ORCID: Hyun Woo Bae (http://orcid.org/0000-0002-6445-3362); Hyundong Kim (http://orcid.org/0000-0002-7984-7065); Sung won Choi (http:// orcid.org/0000-0002-2043-4565); Nami Han (http://orcid.org/0000-0003-3651-9755); Mi Ja Eom (http://orcid.org/0000-0002-3652-045X).

(c) This is an open-access article distributed under the terms of the Creative Commons Attribution Non-Commercial License (http://creativecommons.org/ licenses/by-nc/4.0) which permits unrestricted noncommercial use, distribution, and reproduction in any medium, provided the original work is properly cited. Copyright (c) 2016 by Korean Academy of Rehabilitation Medicine 
International Prognostic Scoring System score was 3 and classified as high risk group. Following induction chemotherapy with azacitidine until the 16th cycle, the patient achieved complete remission. Chromosome analysis showed hyperdiploid clone with complex karyotypic abnormalities including trisomy $8(55, \mathrm{XX},+1,+4, \operatorname{del}(5)$ (q13), $+6,+8,+9,+10,+14,+21,+22[17] / 46, \mathrm{XX}[3]$ ).

The patient had been diagnosed with diabetes mellitus (DM) 2 years previously. Serum glucose level was controlled without complications such as diabetic retinopathy or nephropathy including microalbuminuria.

Laboratory test revealed pancytopenia (hemoglobin concentration, $6.0 \mathrm{~g} / \mathrm{dL}$; white blood cell count, $2.25 \times 10^{9}$ / L; platelet count, $12 \times 10^{9} / \mathrm{L}$ ); slightly abnormal serum lipid profile (total cholesterol, $213 \mathrm{mg} / \mathrm{dL}$; high-density lipoprotein, $26 \mathrm{mg} / \mathrm{dL}$; triglyceride, $284 \mathrm{mg} / \mathrm{dL}$ ); normal prothrombin time and activated partial thromboplastin time; and elevated levels of fibrinogen, fibrin degradation product and D-dimer. Thrombolytic therapy and antiplatelet drugs were contraindicated due to thrombocytopenia. Electrocardiography and echocardiography showed no abnormal result.

On physical examination, the patient was alert and hemodynamically stable. Her initial National Institutes of Health Stroke Scale (NIHSS) score was 6 (facial palsy 1 , right arm motor 4 , dysarthria 1 ). Brain magnetic resonance imaging (MRI) revealed scattered acute infarction in the left cerebral deep white matter near primary motor cortex-white matter junction, corona radiata, and angular gyrus. Brain magnetic resonance angiography indicated severe stenosis and/or acute thrombosis of left proximal M1. And, perfusion MRI showed abnormal perfusion with delayed mean transit time (Figs. 1-3).

On the third day of admission, she underwent rehabilitation consult. Manual muscle strength test (MMT) of right arm was grade 1 proximally and grade 0 at wrist; and the strength of right leg was grade 3 at whole lower limb. The patient scored 40 on the Korean version of Modified Barthel Index, suggesting total dependency of activities of daily living (ADL), and exhibited anomic aphasia. On the 27th day of admission, the patient was unable to open her right eye. The result of follow-up
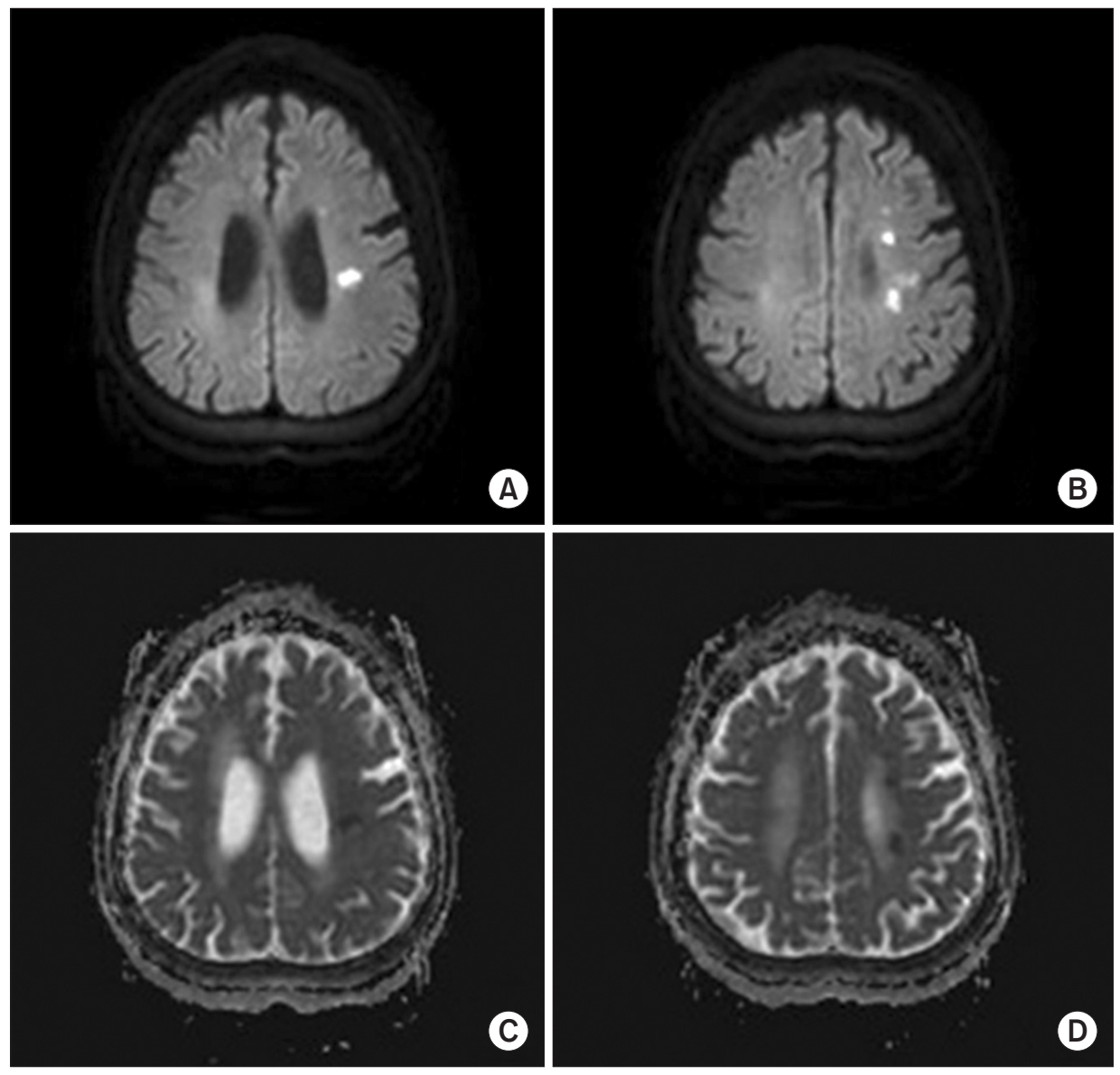

Fig. 1. Acute cerebral infarction in the left cerebral deep white matter showing (A, B) high signal intensity on diffusion-weighted images and $(C, D)$ low signal intensity on apparent diffusion coefficient map of brain magnetic resonance imaging. 

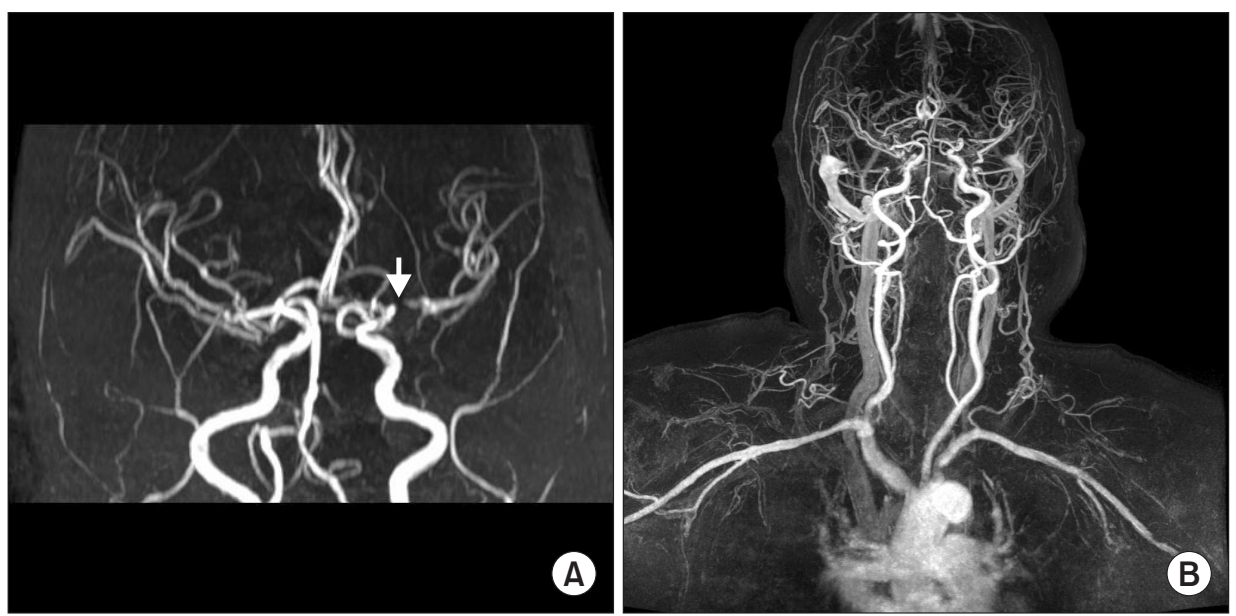

Fig. 2. Images of brain and neck magnetic resonance angiography. (A) A segmental signal loss at the left proximal M1 branch (arrow) suggesting severe stenosis and/ or acute thrombosis. (B) Common carotid artery, internal carotid artery, basilar artery, and vertebral artery show no abnormal vascular finding.
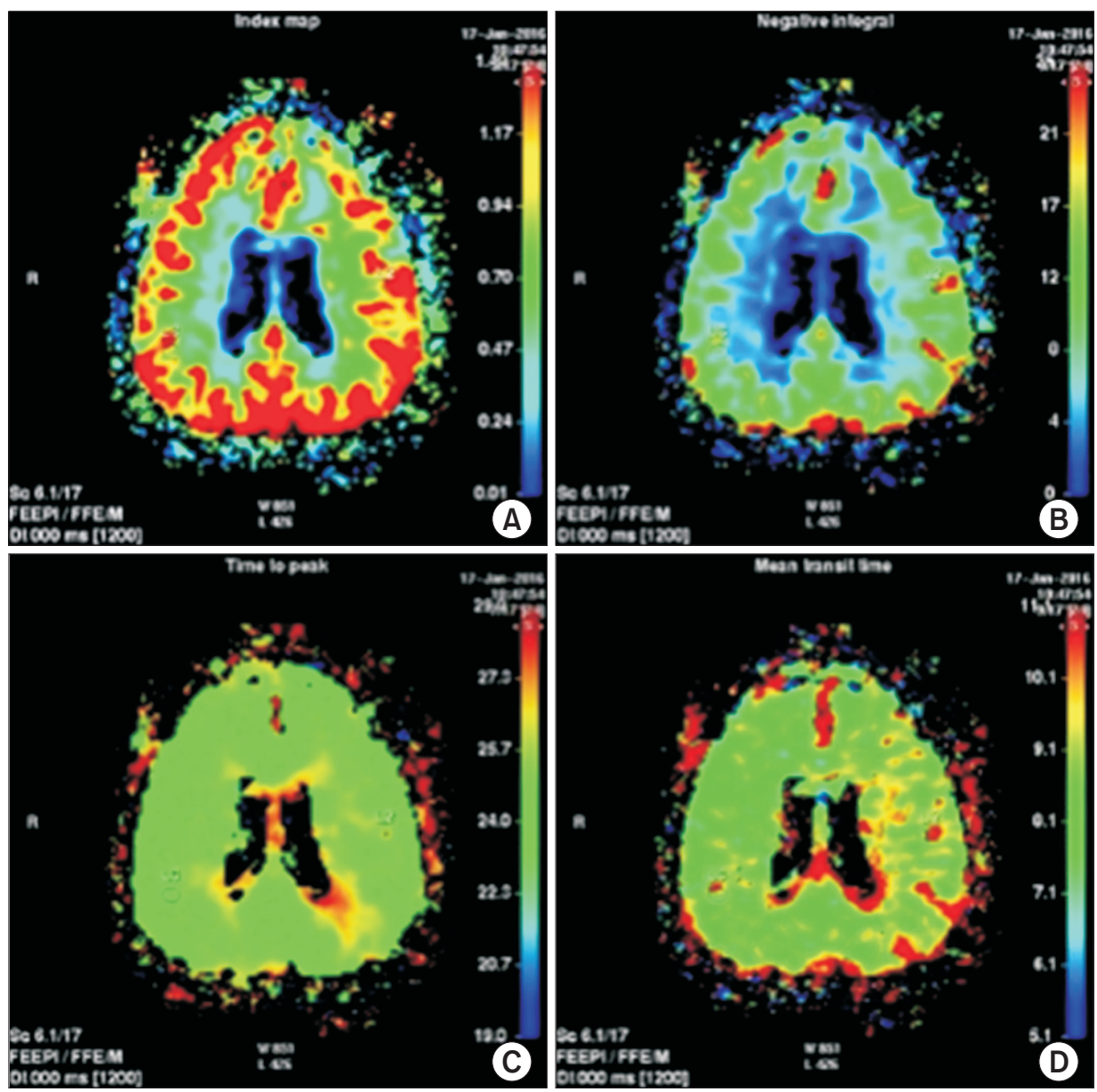

Fig. 3. Perfusion magnetic resonance images of brain. Abnormal perfusion with delayed mean transit time at left deep white matter.

MMT was grade 0 at right whole upper limb, grade 1 at right hip and knee, and grade 2 at right ankle. Further radiologic study was not done despite possibility of progression. During the hospitalization period, conservative treatments alone were administered including intravenous hydration, transfusion, antibiotics for the recurrent infection, and bedside rehabilitative therapy. Additional chemotherapy was not possible because of poor general condition. On the 44th day of admission, the patient was transferred to a secondary referral hospital. On the day of discharge, her NIHSS score was 10 (facial palsy 2, right arm motor 3 , right leg motor 2 , limb ataxia 1 , sensory 1 , 
dysarthria 1), which was increased from that on admission.

\section{DISCUSSION}

MDS is often associated with thrombocytopenia, hence, patients have risk of bleeding. Thrombotic events are rare, especially arterial thrombosis. A cohort of 5,673 MDS patients showed 212 incidents of DVT [3], and the incidence of arterial thrombosis in MDS was uncertain. However, clinically significant arterial thrombotic complications are possible, as in the present case.

Various factors from interventions for the underlying medical condition that can cause thrombotic events were evaluated. The patient had been treated with azacitidine for MDS, which is reported to have rare side effect of bleeding such as intracerebral hemorrhage, but acute cerebral infarction has not been reported previously [4]. Central venous catheter and red blood cell transfusion are possible risk factors for thrombosis in MDS. Catheterrelated risk is greatest at or immediately following catheterization, and declines rapidly in the subsequent weeks [3]. However, the patient experienced no thrombotic event at the time of insertion and during the following month. Also, transfusion was not administered over the last 1-year period.

Comorbidities are suggested risk factors of arterial thrombosis. Although DM and mild dyslipidemia are risk factors for stroke, they were well controlled in our patient. DM guideline recommends hemoglobin Alc (HbAlC) of $8.5 \%$ for those with major comorbidity, dependent ADL, and multisystem disease, etc. [5]. In this case, HbAlc was $8.4 \%$, within the guideline considering age and comorbid MDS.

In addition, the 10-year stroke probability was $9 \%$ using stroke risk profile based on the Framingham study [6]. Average 10-year stroke probability based on patient's age group was $10.9 \%$. Therefore, the risk of stroke was lower than the average for age. Scattered pattern of infarction often has cardioembolic origin, but electrocardiography and echocardiography of the patient were normal.

These findings suggested that the cerebral arterial infarction in this patient was a rare result of MDS with pancytopenia rather than conventional risk factors for stroke.

Trisomy 8 is reportedly a risk factor for thrombosis in MDS and promotes cytokine production [7]. Also, serum level of thrombopoietic and inflammatory cytokines such as interleukin (IL)-6 and IL-8 are increased in MDS [8]. Another study on genetic expression of early hematopoietic cells from MDS with trisomy 8 indicated overexpression of several immune and inflammatory response genes including IL-6 [9]. Likewise, specific cytogenetic abnormalities may be associated with rare complications of MDS. Thus, the blood vessel injury and thrombosis may have resulted from complex chromosomal abnormality including trisomy 8, abnormal inflammatory process, and as yet unrevealed mechanism(s) in MDS. Guidelines to treat cerebral infarction and concomitant MDS are currently unavailable. Further studies are needed to clarify the pathophysiology including unknown immunophenotype and genetic aberration.

\section{CONFLICT OF INTEREST}

No potential conflict of interest relevant to this article was reported.

\section{REFERENCES}

1. Bennett JM, Kouides PA, Forman SJ. The myelodysplastic syndromes: morphology, risk assessment, and clinical management (2002). Int J Hematol 2002;76 Suppl 2:228-38.

2. Yang X, Brandenburg NA, Freeman J, Salomon ML, Zeldis JB, Knight RD, et al. Venous thromboembolism in myelodysplastic syndrome patients receiving lenalidomide: results from postmarketing surveillance and data mining techniques. Clin Drug Investig 2009;29:161-71.

3. Smith SW, Sato M, Gore SD, Baer MR, Ke X, McNally D, et al. Erythropoiesis-stimulating agents are not associated with increased risk of thrombosis in patients with myelodysplastic syndromes. Haematologica 2012; 97:15-20.

4. DailyMed. VIDAZA: azacitidine injection, powder, lyophilized, for solution [Internet]. Maryland: US National Library of Medicine; c2016 [cited 2016 Nov 12]. Available from: https://dailymed.nlm.nih.gov/dailymed/drugInfo.cfm?setid=3495a71a-cc04-4776-851ff185956f32af.

5. American Diabetes Association. Older adults. Diabetes Care 2016;39(Suppl 1):S81-S85. 
6. D'Agostino RB, Wolf PA, Belanger AJ, Kannel WB. Stroke risk profile: adjustment for antihypertensive medication. The Framingham Study. Stroke 1994;25: 40-3.

7. Kimura S, Kuroda J, Akaogi T, Hayashi H, Kobayashi Y, Kondo M. Trisomy 8 involved in myelodysplastic syndromes as a risk factor for intestinal ulcers and thrombosis: Behcet's syndrome. Leuk Lymphoma 2001;4 2:115-21.

8. Hsu HC, Lee YM, Tsai WH, Jiang ML, Ho CH, Ho CK, et al. Circulating levels of thrombopoietic and inflammatory cytokines in patients with acute myeloblastic leukemia and myelodysplastic syndrome. Oncology 2002;63:64-9.

9. Chen G, Zeng W, Miyazato A, Billings E, Maciejewski JP, Kajigaya $S$, et al. Distinctive gene expression profiles of CD34 cells from patients with myelodysplastic syndrome characterized by specific chromosomal abnormalities. Blood 2004;104:4210-8. 